\title{
Modulating the Reactivity of Electrode Surfaces by Electrostatic Assembly of Metal Nanoparticles and Quantum Dots
}

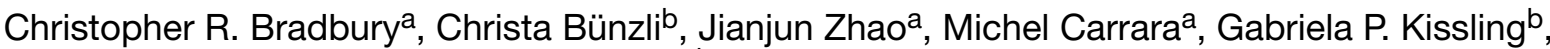 \\ Evren Aslan-Guerela ${ }^{\mathrm{a}}$, and David J. Fermín*b
}

\begin{abstract}
Charge transport phenomena in opto-electronic devices featuring functional polymers and nanostructured materials critically depend on the electronic communication between the building blocks and the metal contacts. The generation of ordered multilayer structures at electrode surfaces is often a key requirement to avoid electrically isolated (inactive) areas in the devices. This issue is particularly crucial in hybrid photovoltaic, light emitting and electrochromic systems. In the present contribution, the properties of electrode surfaces modified by electrostatic layer-by-layer methods are highlighted as a versatile approach for generating two- and three-dimensional assemblies of nanostructures. The connectivity between the nanoparticles and the electrode surface is probed by a variety of techniques including Kelvin probe, electrostatic force microscopy and electrochemical methods. The main characteristics of electrode surfaces modified by electrostatic self-assembly are illustrated with several examples involving metal nanostructures and CdTe quantum dots.
\end{abstract}

Keywords: EFM · Electrochemistry · Electrode surfaces · Electrostatic layer-by-layer assembly · Kelvin probe Metal nanoparticles · Polyelectrolyte $\cdot$ Quantum dots

\section{Introduction}

Over the last ten years, there have been unprecedented advances in the synthesis of functional supramolecular structures, nanostructured materials, redox/conducting polymers and biomolecular components. We have also witnessed important progress in the controlled assembly of these materials at surfaces and their characterisation below single molecule resolution. Towards the incorporation of these materials into devices, the key challenge is to effectively control their electronic communication with metal contacts. Charge transport in hybrid heter-

${ }^{*}$ Correspondence: Dr. D. J. Fermín ${ }^{\mathrm{b}}$

Tel.: +44 1179288981

Fax: +44 1179250612

E-mail: david.fermin@bristol.ac.uk

aDepartement für Chemie und Biochemie

Universität Bern

Freiestrasse 3

$\mathrm{CH}-3012$ Bern

bSchool of Chemistry

University of Bristol

Cantock's Close, Bristol BS8 1TS, UK ostructures often differs from the characteristic high charge mobility through energy bands observed in metal and semiconductor crystals. In fact, the weak electronic coupling between the various functional groups in the assembly manifests itself by hopping type mechanisms through localised energy levels. A recent example of the complexity of charge transport across hybrid heterostructures is given by the controversy over the operating mechanism of the so-called light-emitting electrochemical cells. ${ }^{[1]}$ Our research activities are focused on the characterisation and modulation of the dynamics of charge transport in hybrid multifunctional systems employing electrochemical methods. Some of the key organic and polymeric materials we are employing to modify electrode surfaces are displayed in Fig. 1. These self-assembling molecules play a variety of roles including bridge units (I-III), redox blocking layer (I) and two-dimensional redox active polymer initiator (IV). The polyelectrolytes (V-VIII) allowed us to control the adsorption of colloidal nanostructures, while the redox/conducting polymers (IX-XII) are typically used as charge transporting components in multilayer systems.

One of the most versatile methods to assemble different types of materials at electrode surfaces is the so-called electrostatic layer-by-layer assembly (LBL). ${ }^{[2]}$ This simple approach is based on the adsorption of materials featuring an ionised group. Electrostatic adsorption can be used to assemble metal and semiconductor nanostructures, polyelectrolytes, redox active polymers and charged supramolecular components. LBL also offers control over the number density of the adsorbed material by tuning adsorption time and composition of the solutions. In this paper, we highlight our approach to the study of electronic communication between nanomaterials and electrode surfaces based on hierarchical structures constructed by LBL. The selected examples provide clear evidence that the electrochemical reactivity of modified surfaces can be conveniently tuned by electrostatically assembled nanostructures.

\section{Two- and Three-dimensional Arrays of Metal 'Nanocontacts' at Electrode Surfaces}

The controlled assembly of nanostructures at electrode surfaces requires the formation of compact monolayers or molecular films which act as bridging units. In electrochemical systems, molecular bridges can also be used as barriers towards direct electron transfer between the 


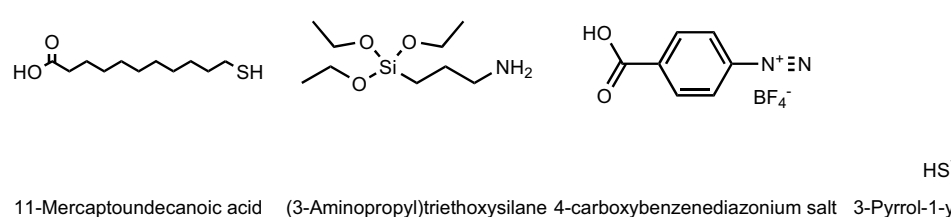

11-Mercaptoundecanoic acid (3-Aminopropyl)triethoxysilane 4-carboxybenzenediazonium salt 3-Pyrrol-1-yl-propane-1-thiol I

II<smiles>CCC(CCCCBr)NC(=O)C(C)(C)C</smiles><smiles>CC(C)(C)CC1C[N+](C)(C)CC1CC(C)(C)C</smiles><smiles>CC1(C)CC2(C)CC1CN2Cl</smiles>

Poly-L-lysine hydrobromide Poly(diallyldimethylammonium Poly(allylamine hydrochloride) V $\mathrm{VI}$
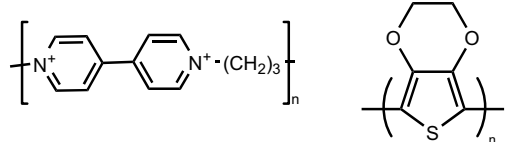

Poly(propyl viologen)

poly(3,4-ethylenedioxythiophene)

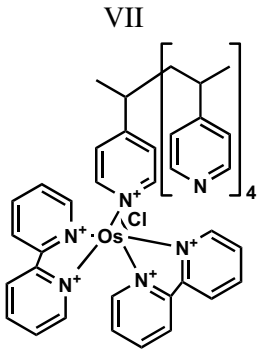

$\left[\mathrm{Os}(\mathrm{bpy})_{2}(\mathrm{PVP})_{5} \mathrm{Cl}\right] \mathrm{Cl}$

XI

Fig. 1. Building blocks and functional materials for the generation of hybrid electroactive systems

Fig. 2. Schematic representation of a three-dimensional array of metal nanoparticles assembled by sequential electrostatic adsorption using a polycation on a $\mathrm{Au}$ surface modified by I

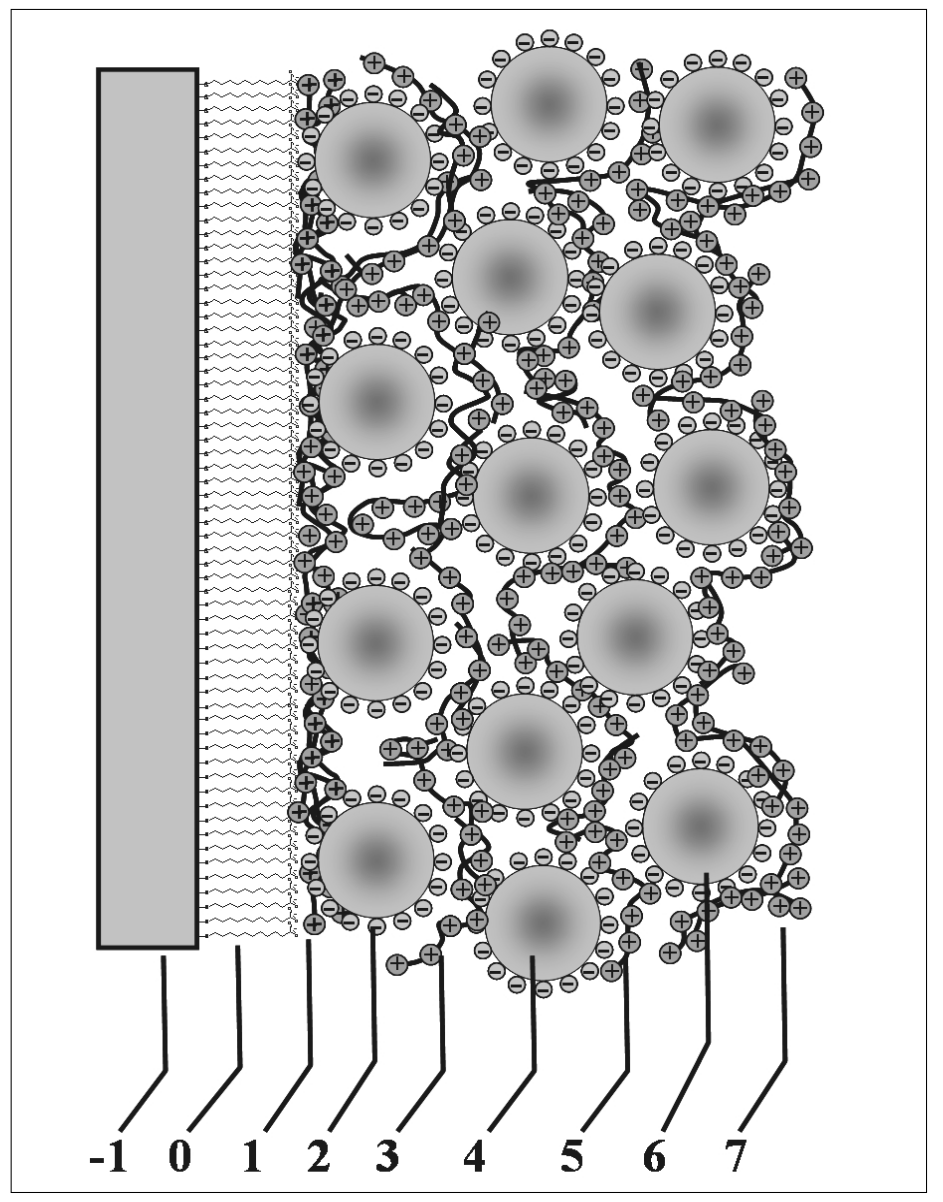

redox probe and the electrode surface. We have employed carboxyl terminated alkane thiols such as mercaptoundecanoic acid (I) for the modification of $\mathrm{Au}$ surfaces. A self-assembled monolayer of $\mathbf{I}$ generates a strong barrier for charge transfer to species such as ferri/ferrocyanide. ${ }^{[3-5]}$ The carboxyl termination of the thiol allows the subsequent electrostatic LBL growth of hybrid architectures featuring polyelectrolytes and metal nanoparticles as schematically illustrated in Fig. 2. ${ }^{[6]}$ This simple approach allows two- or three-dimensional arrays of nanocontacts with tunable electrocatalytic properties to be created. In addition, the energetics of charge injection in optoelectronic devices can also be optimised by adsorbing nanostructures with the appropriate work function.

Fig. 3A shows the changes in the work function $(\Phi)$ of a $\mathrm{Au}$ surface during the LBL growth of 3D assemblies of Ag@Au core-shell nanoparticles and poly-L-lysine (V). The nanoparticles were obtained by the reduction of $\mathrm{Ag}$ on $\mathrm{Au}$ cores of $16 \mathrm{~nm}$ diameter in the presence of citrate. ${ }^{[8]}$ In these experiments, the effect of each layer is investigated on a freshly prepared surface as reported previously. ${ }^{[6,7]}$ Kelvin probe measurements were performed in a dry glove box under inert atmosphere. Point 0 in Fig. $3 \mathrm{~A}$ corresponds to the work function of the Au surface modified by a self-assembled monolayer of I. The oriented dipoles associated with the thiol molecules generate a $0.3 \mathrm{eV}$ increase of $\Phi$. The electrostatic adsorption of $\mathbf{V}$ on the carboxyl terminated thiol generates an overcompensation of the surface charge, leading to a $0.5 \mathrm{eV}$ decrease in $\Phi$. The adsorption of the metal nanoparticles generates an increase in $\Phi$ as a result of the equilibration of the Fermi level in the particle and the metal electrode. Consequently, the values obtained for the metal nanoparticle terminated assemblies (even layer numbers) are associated with the $\Phi$ of the metal nanostructures.

The dependence of $\Phi$ on the mole fraction of $\mathrm{Ag}$ in the core-shell structure is shown in Fig. 3B. The increase in the Ag mole fraction from 0.1 to 0.5 involves a linear increase in the average shell thickness from 0.4 to $2 \mathrm{~nm} .{ }^{[8]}$ Although TEM and UV-visible spectroscopy provide strong evidence of the core-shell geometry of these particles, the changes in $\Phi$ are rather small for Ag shell thicknesses below $1 \mathrm{~nm}$. This result is somewhat surprising, given that the most commonly cited value of $\Phi$ for bulk polycrystalline $\mathrm{Ag}$ is $4.50 \mathrm{eV}$, ${ }^{[9]}$ while for $\mathrm{Au}$ it is $5.20 \mathrm{eV}$ (see point -1 in Fig. 3A). The Au-like work function of these coreshell nanostructures can be associated with the presence of a thin oxide layer at the silver surface. Our previous studies have shown that Ag nanoparticles synthesised following similar protocols exhibited a sig- 

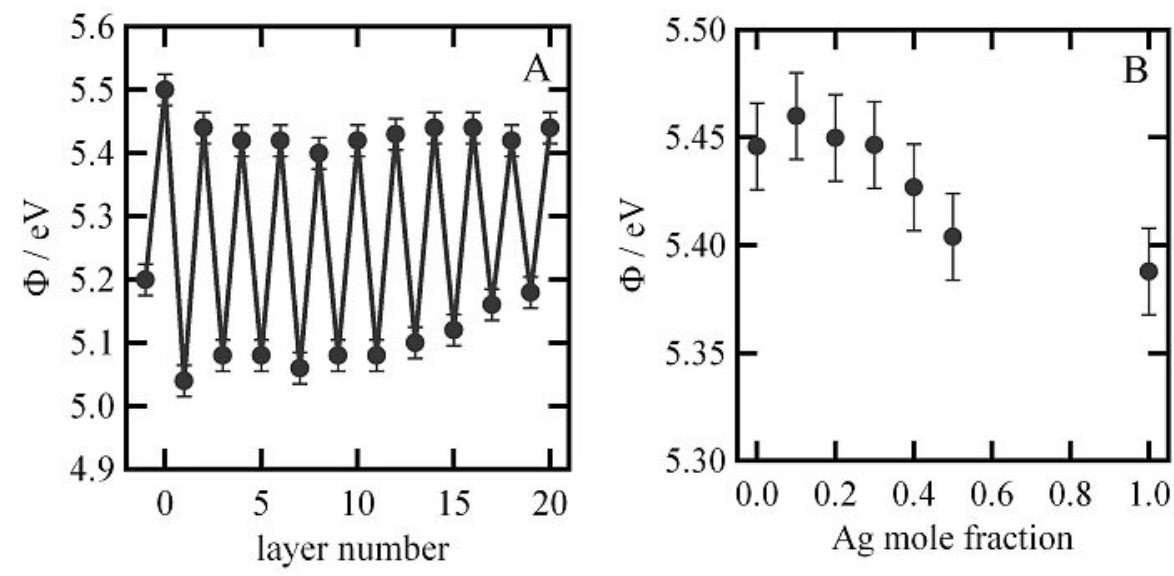

Fig. 3. Modulation of the electrode work function upon the LBL growth of a three dimensiona assembly based on $\mathbf{V}$ and $\mathrm{Ag} @$ Au nanostructures with 0.4 mole fraction of $\mathrm{Ag}(\mathrm{A})$. As indicated in Fig. 2, layer number -1 corresponds to the clean Au surface and 0 represents modification by $\mathbf{I}$. Even layer numbers correspond to the nanoparticle terminated surface while odd numbers identify films terminated with $\mathbf{V}$. Work function dependence on the Ag mole fraction in Ag@Au core-shell structures (B). The value corresponding to $100 \% \mathrm{Ag}$ was taken from the analysis of $7 \mathrm{~nm} \mathrm{Ag}$ nanoparticles. ${ }^{[7]}$

nificant dependence of $\Phi$ on the average particle diameter. ${ }^{[7]}$ High-resolution XPS measurements showed that an oxide layer is present in these particles and the extent of oxygen bonding is affected by the size of the particles. Fig. 3B also shows the average $\Phi$ for a $7 \mathrm{~nm} \mathrm{Ag}$ particle $(100 \%$ mole fraction). It can be seen that the Ag-coated $\mathrm{Au}$ particles approach the $\Phi$ value of $\mathrm{Ag}$ particles with these dimensions. Another aspect affecting the work function of these materials is the citrate layer at the particle surface. Experiments performed with $\mathrm{Au}$ and $\mathrm{Pt}$ nanoparticles have shown that the adsorbed citrate generates a surface dipole which increases the work function by approximately $0.2 \mathrm{eV}$.[6]

Local information on the 'electrical connectivity' between the metal nanoparticles and the electrode surface can be illustrated by electrostatic force microscopy (EFM). ${ }^{[10]}$ In this technique, the phase image of an os- cillating AFM tip is used for mapping the gradient of the electrostatic forces across a surface. The EFM measurements reported herein were performed with a Molecular Imaging Pico LE instrument in Acoustic Mode with Pt coated tips (Nanosensor ${ }^{\mathrm{TM}}$ PPP-NCHPt). The AFM tip is grounded and the voltage bias is defined as sample $v s$. tip. Experiments were conducted in a drybox purged with argon with less than $2 \%$ relative humidity.

The EFM images were recorded in two steps: i) topography and phase images in conventional acoustic AFM mode followed by ii) scanning the surface a second time with the tip lifted away from the surface. This process is schematically illustrated in Fig. 4A. Lifting the AFM tip minimises the contribution from local visco-elastic properties of the system, therefore the observed phase lag of the oscillating tip is mainly induced by electrostatic forces.
Topographic and phase images of an electrostatic assembly of $19 \mathrm{~nm}$ Au nanoparticles before and after the tip lift, respectively are shown in Fig. 4B-C respectively. The sample corresponds to an Au surface sequentially modified by a monolayer of $\mathbf{I}$, a polyelectrolyte multilayer (PEM) comprising of ten bilayers of V and VIII and a sub-monolayer of Au nanoparticles. The nanoparticle coverage was deliberately kept low to avoid the convolution of several particles in the EFM images. The topography (Fig. 4B) shows that the PEM surface is homogeneous with a very low corrugation. After the tip is lifted by 20 $\mathrm{nm}$, the phase image (Fig. 4C) shows only faint features under non-biased conditions. The image in Fig. 4C can be taken as a baseline for the EFM measurements, confirming that electrostatic effects on the tip oscillations are minimal in the absence of an externally applied bias voltage.

The effect of the bias voltage on the phase image is illustrated in Fig. 5. It is observed that the contrast in the EFM phase image increases with increasing bias voltage. In addition, the cross sections also show that the magnitude of the phase shift increases not only in the areas covered by the nanoparticles but over the entire surface. The voltage dependence of the phase shift averaged over an ensemble of eleven particles as well as a section of the PEM film is shown in Fig. 6. The parabolic shape of the EFM signal around $0 \mathrm{~V}$ is characteristic of the potential dependence $\left(\Delta V_{\text {bias }}\right)$ of the electrostatic force $\left(F_{\text {elec }}\right)$,

$$
F_{\text {elec }}=\frac{1}{2} \frac{\partial C}{\partial z} \Delta V_{\text {bias }}^{2}
$$

where $\partial C / \partial z$ corresponds to the derivative of the capacitance and geometric factors with respect to the tip-substrate distance. The stronger dependence of the phase shift on the voltage in nanoparticle covered areas confirms their electrical coupling to
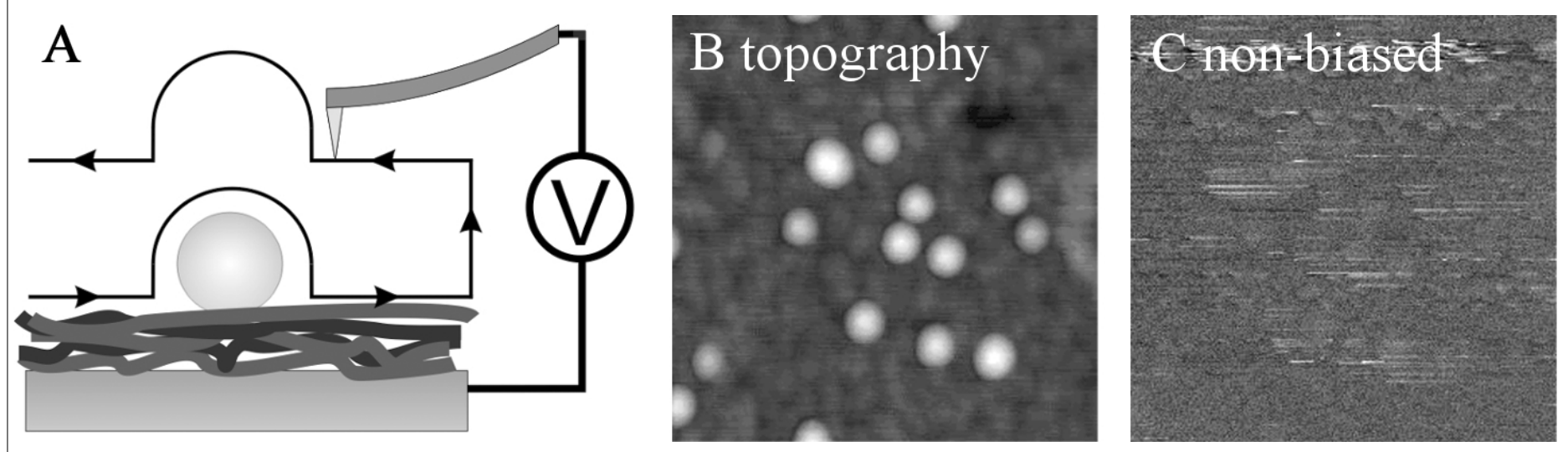

Fig. 4. Schematic image of an EFM measurement (A). The AFM tip initially scans the surface in order to map topographic features, followed by lifting and reverse scanning under an applied bias voltage. The connection between the tip and the substrate represents the bias voltage applied. Topographic $(1000 \mathrm{~nm} \times 1000 \mathrm{~nm} \times 30 \mathrm{~nm})$ image of the forward scan of $19 \mathrm{~nm}$ Au nanoparticles assembled on an Au surface sequentially modified by I and ten bilayers of V/VIII (B). The reverse phase image obtained after lifting the tip by $20 \mathrm{~nm}$ in the absence of an applied bias potential (C). 

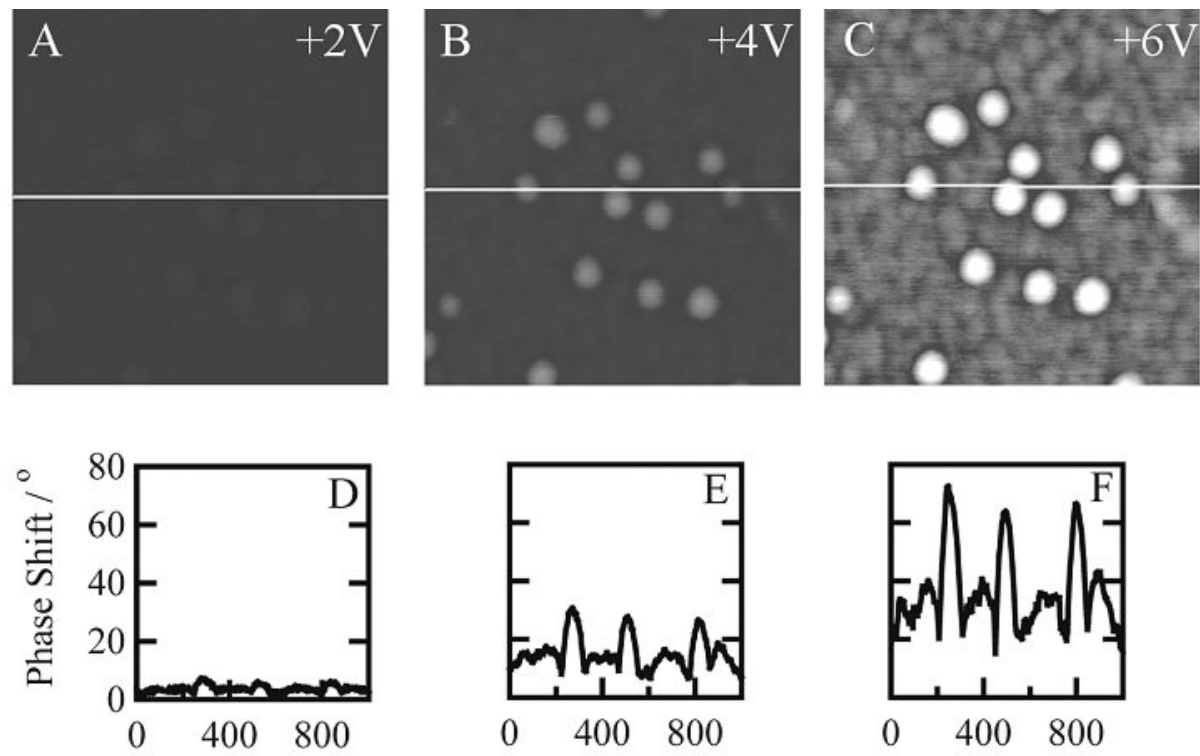

Fig. 5. EFM phase image $(1000 \times 1000 \mathrm{~nm})$ with a $20 \mathrm{~nm}$ lift at $+2(\mathrm{~A}),+4(\mathrm{~B})$ and $+6 \mathrm{~V}$ vs. tip (C) All EFM phase images were scaled to a phase shift of $90^{\circ}$ for the $z$ axis. The line scans in $(A)$ to $(C)$ represent the cross sections shown in (D), (E) and (F), respectively.

the electrode surface. This approach is a useful qualitative tool for examining complex hybrid heterostructures incorporating insulating and metal nanostructures. For instance, the EFM signal of the particles in Fig. 6 shows that the Fermi level in each individual particle is shifted with respect to the Fermi level of the tip. This behaviour indicates that the metal nanoparticles are electrically connected to the electrode surface. However, it should be clarified that information on the conductivity of the system is not directly accessible from these measurements as no current flows between the tip and the substrate. Further

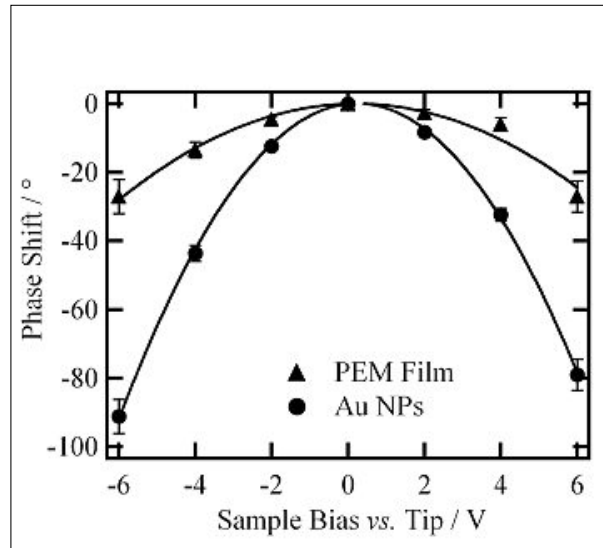

Fig. 6. EFM phase shift of the PEM film and the Au NPs with increasing bias voltage. The EFM response is averaged over eleven $\mathrm{Au}$ particles. The EFM signal associated with the PEM film was measured from averaging the change in phase shift in areas not covered by nanoparticles. The continuous lines correspond to a least-square fit to the square of the applied bias. quantitative information can be obtained by adding an $\mathrm{AC}$-voltage across the tip and the substrate. ${ }^{[11]}$ Brus and co-workers have demonstrated that measuring the first and second harmonic signal of the AC-voltage allows estimating parameters such as local polarisability and charge distribution across nanostructured surfaces. ${ }^{[12]}$

\section{Two-dimensional Arrays of Nanoelectrodes}

The strong connectivity between metal nanoparticles electrostatically adsorbed on electrode surfaces allows us to modulate the electrochemical reactivity of electrodes. This approach is exemplified by the electrochemical responses of two-dimensional assemblies of $19 \mathrm{~nm} \mathrm{Au}$ and $14 \mathrm{~nm}$ Pt nanoparticles at modified indium-doped tin oxide (ITO) electrodes, as shown in Fig. 7. The ITO electrodes are initially modified by a monolayer of (3-aminopropyl)triethoxysilane (II), which allows electrostatic adsorption of the citrate stabilised nanoparticles. The voltammetric signals recorded in diluted $\mathrm{HClO}_{4}$ solutions are contrasted with the responses obtained from bulk polycrystalline electrodes. Further details on the nanoparticle synthesis and electrode preparation are reported elsewhere. [13]

The voltammogram of the $\mathrm{Au}$ nanoassembly in Fig. 7A exhibits the same characteristic oxide formation and reduction waves as the bulk Au electrode. The magnitude of the response is approximately ten times smaller than for the bulk electrode, which is consistent with the estimated particle number density of $2.6 \times 10^{10} \mathrm{~cm}^{-2}$ obtained from SEM analysis (not shown). It is also noted that the shape of the oxide formation appears somewhat different for the two electrodes. These differences can be rationalised in terms of variations in the density of ordered crystal facets.

In the case of the $14 \mathrm{~nm}$ Pt assembly, the electrochemical response exhibited more significant differences with respect to the bulk polycrystalline electrode (Fig. 7B). The hydrogen adsorption-desorption peaks observed between -0.1 and $-0.3 \mathrm{~V} v s$. Ag/ $\mathrm{AgCl}$ appear suppressed in the nanoparticle assembly. This effect can arise due to the blocking of $\mathrm{H}$-adsorption sites by the stabilising citrate molecules. In addition, the

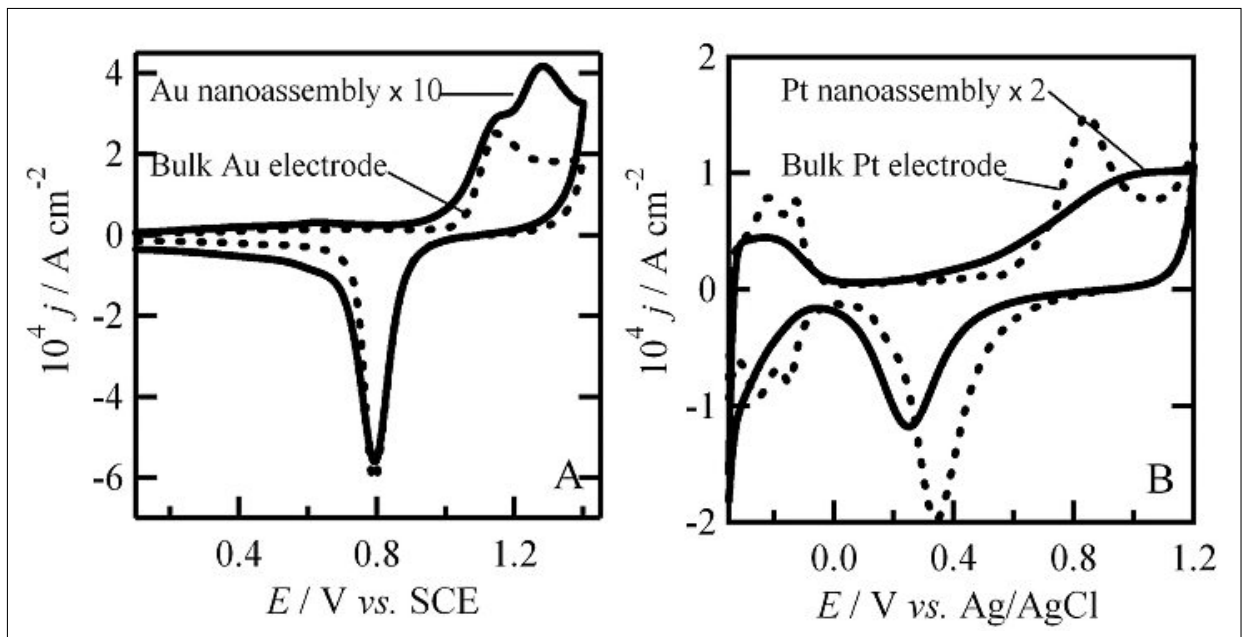

Fig. 7. Cyclic voltammogram of $19 \mathrm{~nm}$ Au nanoparticles (solid line) electrostatically adsorbed on an ITO electrode modified with II (A). The voltammogram was recorded in $0.01 \mathrm{~mol} \mathrm{dm}^{-3} \mathrm{HClO}_{4}$ at $100 \mathrm{mV} \mathrm{s}^{-1}$. The response is compared to the voltammogram of a bulk polycrystalline Au electrode (dashed line). Cyclic voltammogram of a $14 \mathrm{~nm}$ Pt nanoassembly (solid line) at $50 \mathrm{mV} \mathrm{s}^{-1}$ in $1 \times 10^{-3}$ mol dm$~^{-3} \mathrm{HClO}_{4}$ and $0.01 \mathrm{~mol} \mathrm{dm}^{-3} \mathrm{NaClO}_{4}(\mathrm{~B})$. The response obtained with a polycrystalline $\mathrm{Pt}$ electrode (dashed line) under identical conditions is also illustrated. The currents associated with the metal nanoparticle assemblies were multiplied by the indicated factors to facilitate qualitative comparison with the corresponding polycrystalline electrodes. 
peaks associated with oxide formation and reduction are broader and slightly shifted with respect to the response at the bulk electrode. The suppressed $\mathrm{H}$ desorption signal in the cyclic voltammograms could suggest that the electrocatalytic activity of the $\mathrm{Pt}$ nanoparticles towards hydrogen evolution is lower than for bulk Pt. However, our preliminary studies based on scanning electrochemical microscopy have shown that the average particle reactivity is significantly larger than for polycrystalline Pt electrodes. We are currently investigating the structural aspects of the nanoparticle surface, employing surface sensitive electrochemical probes as recently developed by Feliu and co-workers. ${ }^{[14]}$

\section{Quantum Dot-modified Electrodes}

Self-assembled layers of quantum dots (QDs) are a highly promising element in the development of novel optoelectronic devices. The extraordinary luminescent behaviour of QDs has been well characterised and they are already used in applications such as photovoltaics ${ }^{[15,16]}$ and LEDs. ${ }^{[17,18]}$ Towards the development of novel hybrid devices, we are currently investigating the charge transport properties of QD assemblies at electrode surfaces employing electrochemical techniques.

Following the modification strategies previously highlighted, Au electrodes were sequentially modified by self-assembled monolayers of $\mathbf{I}$, followed by an ultrathin film of poly(diallyldimethylammonium chloride) (VI) and a monolayer of mercaptopropionic acid stabilised CdTe QDs, prepared following the method presented

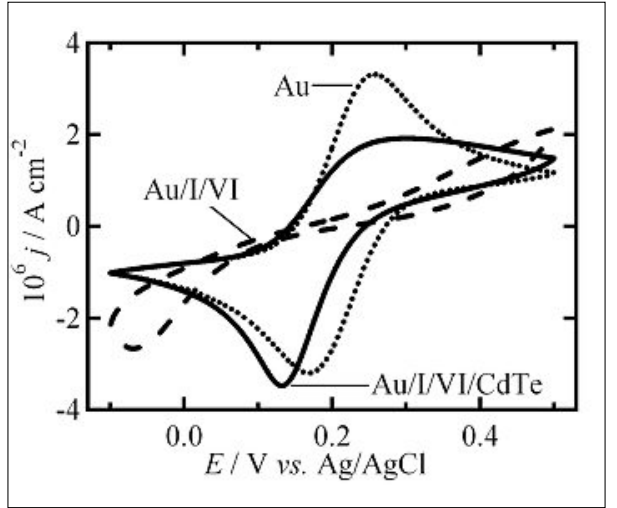

Fig. 8. Cyclic voltammogram of a $3.7 \mathrm{~nm}$ CdTe dot assembly (solid line) in the presence of an equimolar concentration of the ferri/ ferrocyanide redox couple $\left(1 \times 10^{-3} \mathrm{~mol} \mathrm{dm}^{-3}\right)$. The voltammetry is compared to that of a clean (dotted line) and a modified (dashed line) Au electrode. The electrode modification consisted of a blocking self-assembled monolayer of $I$ and an ultrathin film of VI. The voltammograms were recorded at $100 \mathrm{mV} \mathrm{s}^{-1}$.
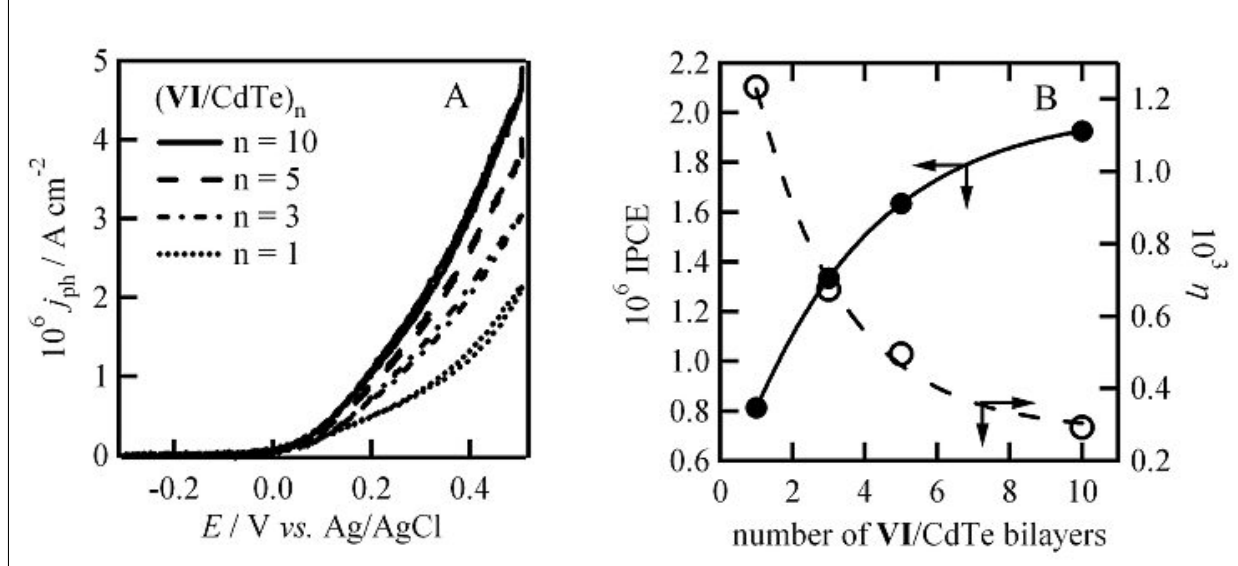

Fig. 9. Photocurrent vs. potential curves for different numbers of VI/CdTe bilayers on ITO recorded under illumination with a photon flux density of $6.4 \times 10^{18} \mathrm{~cm}^{-2} \mathrm{~s}^{-1}$ in $0.1 \mathrm{~mol} \mathrm{dm}^{-3} \mathrm{Na}_{2} \mathrm{SO}_{3}(\mathrm{~A})$. Dependence of IPCE (solid line) and $\eta$ (dashed line) on the number of bilayers calculated from data in $(\mathrm{A})$ at $0.3 \mathrm{~V}$ vs. $\mathrm{Ag} / \mathrm{AgCl}(\mathrm{B})$.

by Zhang and Yang. ${ }^{[19]}$ The voltammetric response in the presence of the ferri/ferrocyanide redox couple for the CdTe nanoassembly is contrasted to the clean and modified electrode in Fig. 8. The I/VI film generates a blocking layer at the electrode surface, strongly suppressing the faradaic current as observed in $\mathbf{I} / \mathbf{V}$ films. ${ }^{[3]}$

The electrostatic adsorption of the 3.7 nm CdTe QDs brings about a significant increase of the faradaic current. An interesting observation is the more pronounced reduction peak. This behaviour is significantly different from our previous studies employing Au nanoparticles, in which the oxidation and reduction rates are equally increased. ${ }^{[3]}$ Other studies employing insulating $\mathrm{SiO}_{2}$ nanoparticles clearly demonstrated that this behaviour is not connected to the generation of defects in the ultrathin film by the adsorbed nanostructures. ${ }^{[4]}$ The unique rectifying properties of the $\mathrm{CdTe}$ QDs arise from the density of states in the dots at the redox energy of the ferri/ferrocyanide couple. Recent investigations have shown that the valence band edge of these dots is located at $0.29 \mathrm{~V} v s$. $\mathrm{Ag} / \mathrm{AgCl} .{ }^{[20]}$ The QD mediated oxidation of ferrocyanide is inhibited by the mismatch of the energy levels of the redox probe with the density of states of the particles. The occupied energy levels of ferrocyanide overlap only with the fully occupied valence band of the dots. Thus, a hole-injection step from the electrode to the valance band is required prior to the ferrocyanide oxidation. As the edge of the valance band is below the redox energy of the probe, the hole injection step generates an overpotential for the oxidation reaction. On the other hand, the reduction of ferricyanide requires a lower overpotential due to the overlap between the unoccupied energy level of the ion and the valence band of the QDs.

\section{Photoelectrochemical Response of Quantum Dot-modified Electrodes}

Electrostatic LBL methods allow the construction of three dimensional arrays of CdTe QDs, as schematically illustrated in Fig. 2. Multilayers of CdTe/VI assembled on ITO generate photoelectrochemical responses in the presence of $\mathrm{SO}_{3}{ }^{2-}$ upon photoexcitation of the dots as exemplified in Fig. 9A. Details on the electrode preparation can be found elsewhere. [21] The mechanism behind the photocurrent response involves the hole capture at the valence band edge by the $\mathrm{SO}_{3}{ }^{2-}$ ion, followed by the transport of the electrons to the electrode via the conduction band of the dots. It can be observed that the photocurrent responses increase with increasing number of $\mathrm{CdTe} / \mathrm{VI}$ bilayers. Furthermore, the photocurrent responses in Fig. 9A are characterised by a strong dependence on the potential bias. This behaviour is indicative of recombination losses in the interfacial region. Considering the irreversible nature of the $\mathrm{SO}_{3}{ }^{2-}$ oxidation, the most probable recombination path involves electron injection from the electrode to the valence band holes. As the electrode potential is increased, the driving force for electron injection into holes at the valance band edge decreases.

The incident photon-to-current efficiency (IPCE) and the quantum efficiency $(\eta)$ as a function of the number of bilayers are shown in Fig. 9B. The values were calculated from data recorded at $0.3 \mathrm{Vvs}$. Ag/ $\mathrm{AgCl}$. The former parameter is determined by the ratio between the flux of photons and the measured photocurrent, while the latter is defined in terms of flux of photons absorbed by the CdTe multilayer. The IPCE increases with the number of QD layers as the number of excited QDs which can pro- 
vide electrons for photocurrent generation becomes larger. However, the quantum efficiency decreases with increasing QD layer thickness. As the electrolyte penetrates the LBL assembly and the incident photon flux can be considered homogenous due to the low capture cross section, it can be assumed that photogenerated holes are scavenged across the entire film. Consequently, the decrease in the quantum efficiency is assigned to inefficient electron collection from the outer layers. The electrons either react with a species in solution or recombine with the hole in the VB before they reach the ITO electrode.

\section{Conclusions}

The controlled assembly of metal and semiconductor nanostructures can generate highly significant changes in the electrochemical reactivity of electrode surfaces. We have shown that electrostatic layer-bylayer assembly is a convenient and versatile tool to generate a wide range of hierarchical structures. For instance, blocking the electrode surface with an insulating selfassembled monolayer, followed by electrostatic adsorption of the nanostructures allows channelling the transport of charges to the electrode via these 'nanocontacts'. This observation opens the possibility of generating electrode materials in which the work function can be modified in a range of several $\mathrm{eV}$. In addition, the energetics of the charge transport can be finely controlled by adsorbing semiconductor nanostructures.

Another important area in this field is the manipulation of the electrocatalytic activity of the interface. We are currently investigating the reactivity of a variety of metal nanostructures and alloys at modi- fied electrodes employing surface-sensitive electrochemical reactions. The oxidation of small organic molecules such as carbon monoxide and specifically adsorbed metal adatoms can provide useful information on the relationship between reactivity, size and shape of the nanomaterials. ${ }^{[14]}$ Finally, we want to emphasise that these investigations are particularly relevant to sustainable solar energy conversion systems. The construction of low-cost photovoltaic devices based on roll-to-roll technologies is highly compatible with some of the methods described here. In addition, nanoscale materials for efficient hydrogen evolution can provide a viable alternative for chemical energy storage.

\section{Acknowledgements}

The authors are grateful to the valuable contributions of the following people to this work: Dr. Jean-Pierre Abid (EPFL, Lausanne), Prof. Libbero Zupirolli (EPFL, Lausanne), Prof. Hubert Girault (EPFL, Lausanne), Mathias Schnippering (University of Warwick), Dr. Neil Wilson (University of Warwick), Adrian Schindler (Universität Bern), Prof. Thomas Feurer (Universität Bern), Beat Locher (Universität Bern) and Louis Pacheco (Scientec, Les Ulis). The Swiss National Science Foundation (projects: PP002--68708, PP002-$116898 / 1$ and 200021--105238), the PortlandZementstiftung and the Stiftung zur Förderung der Wissenschaftlichen Forschung an der Universität Bern are gratefully acknowledged for their financial support.

Received: May 17, 2008

[1] Q. Pei, A. J. Heeger, Nature Mat. 2008, 7, 167.

[2] G. Decher, Science 1997, 277, 1232.

[3] J. Zhao, C. R. Bradbury, S. Huclova, I. Potapova, M. Carrara, D. J. Fermin, J. Phys. Chem. B 2005, 109, 22985.
[4] J. Zhao, M. Wasem, C. R. Bradbury, D. J. Fermin, J. Phys. Chem. C, 2008, 112, 7284.

[5] C. R. Bradbury, J. Zhao, D. J. Fermin, J. Phys. Chem. C, 2008, 112, 10153.

[6] M. Carrara, J. J. Kakkassery, J.-P. Abid, D. J. Fermin, ChemPhysChem 2004, 5 , 571.

[7] M. Schnippering, M. Carrara, A. Foelske, R. Kötz, D. J. Fermin, Phys. Chem. Chem. Phys. 2007, 9, 725.

[8] J.-P. Abid, PhD Thesis, Swiss Federal Institute of Technology Lausanne, 2003

[9] See articles in 'Silber', Ed. Gmelin, Verlag Chemie, Weinheim, 1970.

[10] D. A. Bonnell, 'Scanning Probe Microscopy And Spectroscopy: Theory, Techniques and Applications', Wiley, New York, 2000.

[11] R. M. Nyffenegger, R. M. Penner, Appl. Phys. Lett. 1997, 71, 1878.

[12] O. Cherniavskaya, L. Chen, V. Weng, L. Yuditsky, L. E. Brus, J. Phys. Chem. B 2003, 107, 1525.

[13] J. Zhao, PhD Thesis, University of Bern, 2008.

[14] J. Solla-Gullon, P. Rodriguez, E. Herrero, A. Aldaz, J. M. Feliu, Phys. Chem. Chem. Phys. 2008, 10, 1359.

[15] N. A. Kotov, I. Dekany, J. H. Fendler, J. Phys. Chem. 1995, 99, 13065.

[16] A. Hagfeldt, M. Grätzel, Chem. Rev. 1995, $95,49$.

[17] S. Coe, W. K. Woo, M. Bawendi, V. Bulovic, Nature 2002, 420, 800.

[18] J. G. Pagan, E. B. Stokes, K. Patel, C. C. Burkhart, M. T. Ahrens, P. T. Barletta, M. O'Steen, Solid-State Electronics 2006, 50, 1461.

[19] H. Zhang, B. Yang, Thin Solid Films 2002, $418,169$.

[20] G. P. Kissling, Diploma Dissertation, University of Bern, 2007.

[21] C. Bünzli, Diploma Dissertation, University of Bern, 2007. 